\title{
Expression of phenotypic markers of mast cells, macrophages and dendritic cells in gallbladder mucosa with calculous cholecystitis
}

\author{
Aldona Anna Kasprzak ${ }^{1}$, Miroseaw Szmyt ${ }^{1}$, Wojciech Malkowski², Joanna Surdyk-Zasada ${ }^{1}$, \\ Wieseawa Przybyszewska ${ }^{1}$, Jacek Szmeja ${ }^{3}$, Celina Helak-Łapaj ${ }^{4}$, Agnieszka Seraszek-Jaros ${ }^{4}$, \\ ELŻBIETA KACZMAREK ${ }^{4}$
}

\author{
${ }^{1}$ Chair and Department of Histology and Embryology, Poznan University of Medical Sciences, Poland \\ ${ }^{2}$ Chair of General Surgery T. Chałubiński Municipal Hospital, Ostrów Wielkopolski, Poland \\ ${ }^{3}$ Department of General Surgery, Oncologic Gastroenterologic Surgery and Plastic Surgery, \\ Poznan University of Medical Sciences, Poland \\ ${ }^{4}$ Department of Bioinformatics and Computational Biology in the Chair of Clinical Pathomorphology, \\ Poznan University of Medical Sciences, Poland
}

\begin{abstract}
The study aimed at quantitative analysis of expression involving markers of mast cells (tryptase), monocytes/macrophages (CD68 molecule) and dendritic cells (S100 protein) in gallbladder mucosa with acute and chronic calculous cholecystitis.

Routinely prepared tissue material from the patients with acute (ACC) $(n=16)$ and chronic calculous cholecystitis (CCC) $(n=55)$ was evaluated. Three cellular markers were localized by immunocytochemistry. Their expression was quantified using spatial visualization technique.

The expression of tryptase was similar in acute and chronic cholecystitis. CD68 expression in ACC was significantly higher than in the CCC group. Expression of S100 protein was significantly higher in CCC as compared to the ACC group. No significant correlations were disclosed between expression of studied markers and grading in the gallbladder wall. A weak negative correlation was noted between expression of CD68 and number of gallstones in the CCC group.

The spatial visualization technique allowed for a credible quantitative evaluation of expression involving markers of mast cells (MCs), monocytes/macrophages ( $\mathrm{Mo} / \mathrm{Ma})$ and dendritic cells (DCs) in gallbladder mucosa with ACC and CCC. For the first time mucosal expression of S100 protein-positive DCs was evaluated in calculous cholecystitis. The results point to distinct functions of studied cell types in the non-specific immune response in calculous cholecystitis.
\end{abstract}

Key words: cholelithiasis, cholecystitis, tryptase, CD68, S100 protein.

\section{Introduction}

Acute cholecystitis most frequently coexists with cholelithiasis. At the beginning, cells of inflammatory infiltrates include neutrophils, which may lead to a phlegmon. With elapsing time, granulocytes gradually vanish, replaced by lymphocytes, plasma cells and macrophages. Connective tissue proliferates, leading to thickening and stiffening of gallbladder walls [1].
The most frequent morphological alteration in cholelithiasis involves chronic cholecystitis with thickening of the gallbladder wall, steatocholecystitis, muscle hypertrophy and incrustation with gallstones $[1,2]$. Microscopically detectable lesions of mucosa in chronic calculous cholecystitis (CCC) comprise inflammatory infiltrates of a variable cellular composition, present in mucosa and in deeper layers of the gallbladder wall [3]. Several morphological varieties of CCC 
exist. Cholesterolosis is present in $20 \%$ of cholecystectomy specimens with foamy macrophages in lamina propria and epithelium [4]. A special form of CCC showing localized accumulation of abundant foamy macrophages intermingled with lymphoplasmacytic cells (mainly $\mathrm{T}$ cells) is xanthogranulomatous cholecystitis [5-8]. In development of such lesions participation of bacterial antigens, in particular those of $E s$ cherichia coli, is suggested [7, 9]. Mucosa of calculous gallbladder also contains infiltrates of mast cells (MCs) [5]. On the other hand, the so-called eosinophilic cholecystitis ( $\geq 90 \%$ eosinophils) most frequently appears in acalculous cholecystitis [10].

Tissue MCs play a role in allergic reactions, angiogenesis, non-specific immunity and at preliminary stages of the specific immune response of the body $[11,12]$. Their basic categorization in humans reflects their content of neutral serine proteases (tryptase and chymase). Tryptase seems to participate in pro-inflammatory functions of MCs, while chymase seems to be more involved in inflammatory reactions [13]. Studies have demonstrated that MCs may also be involved in synthesis of chemokines and cytokines $[11,14]$. Tryptase-positive and chymase-negative cells in bronchial muscle biopsies were shown to be the source of interleukins (ILs) IL-5 and IL-6, while IL-4 was distributed in both MC phenotypes $[14,15]$. Also an increase in the number of MCs staining for tumor necrosis factor $\alpha$ (TNF- $\alpha$ ) was demonstrated in asthmatic biopsies [14]. TNF- $\alpha$ $\mathrm{mRNA}$ and protein were expressed constitutively in isolated human intestinal MCs [16]. Attempts were made to describe involvement of MCs both in physiology and in several inflammatory diseases and non-inflammatory diseases of the alimentary tract in humans $[12,17,18]$, including pathology of gallbladder [19-22]. Higher numbers of MCs were detected in chronic exacerbated and chronic sclerotic cholangitis [21], and in Hirschsprung's disease [18], as compared with those in controls. An increased number of MCs in lamina propria was demonstrated in symptomatic cholelithiasis, as compared to patients with biliary dyskinesia [22]. The quoted correlations between number of MCs and intensity of inflammation and/or fibrosis in biliary ducts are not unequivocal $[17,21,22]$. In pathogenesis of gallbladder diseases many pathomechanisms with involvement of MCs are considered [5]. Mast cells containing numerous chemotactic factors may be responsible for accumulation of also numerous eosinophils in inflammatory infiltrates, observed in chronic cholecystitis [23] and in eosinophilic gastroenterocolitis [24].

Our earlier studies demonstrated eosinophils as a dominating type of cells in the inflammatory infiltrate in a great number (27\% of cases) of young patients (up to 25 years old), as compared to a single patient in the older group [25].

The role of macrophages in pathogenesis of calculous cholecystitis is only fragmentarily described. Detection and evaluation of their number have taken advantage of various investigatory techniques [5, 19, 20]. Presence of markers typical of tissue (histiocytic) macrophages, particularly presence of $\alpha$-1-antitrypsin and $\alpha-1$-antichymotrypsin, was observed both in normal and nonmetaplastic, chronically inflamed gallbladder mucosa [19]. Some investigators demonstrated significantly more numerous MCs and Mo/Ma in calculous gallbladders with minimal inflammation (classified as "normal") than in gallbladders with features of chronic cholecystitis [5].

Dendritic cells (DCs) are effective antigen-presenting cells that can stimulate both primary and secondary $\mathrm{T}$ - and B-cell responses. Dense infiltrates of S100 protein-positive DCs were noted in the majority of hyperplastic tonsils [26] and Langerhans cell sarcoma that involved gallbladder [27]. Their distribution in gallbladder mucosa and role in calculous cholecystitis have not been studied to date.

This study aimed at quantitative analysis of expression involving tryptase (mast cells), CD68 (monocytes/macrophages) and S100 protein (dendritic cells) in gallbladder mucosa of patients with acute and chronic types of calculous cholecystitis, in order to elucidate their possible differential distribution and roles.

\section{Material and methods}

\section{Patients}

All the patients were subjected to cholecystectomy in the T. Chałubiński Municipal Hospital in Ostrów Wielkopolski. The groups with acute calculous cholecystitis (ACC) ( $n=16$; 7 women and 9 men) and chronic calculous cholecystitis (CCC) $(n=55 ; 44$ women and 11 men) included patients who were diagnosed and subjected to surgery in the period 2003-2007. Duration of cholelithiasis symptoms in the analysed groups of patients most frequently ranged from 6 months to about one year. The available epidemiological data included age, gender and body mass index (BMI) upon admission to the hospital. The other available variables included results of laboratory/biochemical tests. The number of gallstones disclosed following cholecystectomy was estimated using a semi-quantitative 1-4 point scale, as previously described [28]. In both groups of patients mostly mixed, cholesterol/pigment/calcium gallstones were detected. However, their detailed chemical composition was not tested. Written informed consent was obtained from each patient before the operation, and approval for the study was granted by the institution's Ethical Committee (no. 21/09).

\section{Tissue material and microscopy image analysis}

The archival material was fixed in buffered $10 \%$ formalin and embedded in paraffin using the routine procedure, and $\sim 5 \mu \mathrm{m}$ paraffin sections were placed on SuperFrost/Plus microscope slides. Studies using paraf- 
fin sections included staining with haematoxylin and eosin (HE). Patterns of HE-stained gallbladder histological preparations were examined using an Olympus BH-2 light microscope by two histopathologists (WB, AK). Thickness (width) of the total wall of the gallbladder was measured (in $\mathrm{mm}$ ). Each tissue specimen was also evaluated based on a simple numerical scoring system for the grade of lamina propria inflammation (G1) (0-3), and the grade of muscularis externa/adventitia inflammation (G2) (0-3), in which 3 points denoted an intense and, most frequently, diffuse inflammatory infiltrate, 2 points referred to moderately intense but also diffuse inflammatory infiltrate, 1 point indicated individual, dispersed cells or focally arranged cells of inflammatory infiltrate, and 0 points indicated tissue sections in which no cells of inflammatory infiltrate could be detected [28].

\section{Immunocytochemical (IHC) studies}

Detection and studies on cellular localisation of tryptase, CD68 and S100 protein in gallbladder mucosa took advantage of the new polymer-based IHC method [29]. Mouse anti-human monoclonal antibodies (mAbs) were employed, directed against human mast cell tryptase (clone AA1, isotype IgG1) (in dilution 1 : 400) (Code M 7052), against CD68 (in dilution $1: 100)$ and against $\mathrm{S} 100$ protein (in dilution $1: 400$ ) (clone Z0311) (all mAbs from Dako, Glostrup, Denmark). The sections were incubated with these primary $\mathrm{mAbs}$ at night at $4^{\circ} \mathrm{C}$, and afterwards were incubated with dextran backbone to which peroxidase (HRP) was attached and with secondary biotinylated link antirabbit and anti-mouse IgG (Dako REAL ${ }^{\mathrm{TM}}$ EnVision ${ }^{\mathrm{TM}}$ Detection System peroxidase/DAB +, Rabbit/Mouse, Dako). Following deparaffinization and rehydration the preparations were additionally boiled in $10 \mathrm{mM}$ citrate buffer in a $700 \mathrm{~W}$ microwave oven for $18 \mathrm{~min}$ (in the case of anti-tryptase and anti-CD68), washed in phosphate buffered saline (PBS), and then subjected to the reaction according to the standard procedure. Every test was accompanied by a negative control, in which specific antibodies were supplemented by a normal serum of a respective species in $0.05 \mathrm{M}$ Tris- $\mathrm{HCl}, \mathrm{pH} \sim 7.6$, supplemented with $0.1 \%$ bovine serum albumin (BSA) and $15 \mathrm{mM}$ sodium azide. For comparison of MC, $\mathrm{Mo} / \mathrm{Ma}$ and DC marker expression in gallbladder tissues, identical studies were performed in the archival tissue material from reactive lymph nodes $(n=6)$ removed during cholecystectomy (positive control). Studies in the control group aimed only at verification of quality of the applied antibodies and were not subjected to statistical analysis.

Histological slides with expression of all tissue markers were examined under the optical Olympus BH-2 microscope coupled to a digital camera. Colour microscopic images were recorded using the $40 \times$ objec- tive (at least 10 fields in every microscope slide with an IHC positive reaction) and archived using LUCIA Image 5.0 computer software, documenting them in jpg format on the computer hard disc.

\section{Microscopy image analysis and statistical analysis}

The quantitative evaluation of the IHC expression of the proteins was performed using an image processing method based on spatial visualization of markers in microscope images, elaborated and programmed in the A4D computer software C+ + language by Kaczmarek and Strzelczyk [30] and described in detail in our previous paper [31]. Results obtained in the two programs for image analysis (LUCIA Image 5.0 and A4D) were exported to the Microsoft Excel program, and were statistically analysed with the Statistica PL v. 8 program (StatSoft, Inc., Tulsa, OK, USA). In this study results related to expression of proteins were presented as area fraction (percentage) of the IHC reactions manifested by gallbladder mucosa (epithelium + lamina propria).

At the first stage of the statistical analysis, compatibility of the obtained results with a Gaussian distribution was checked using the Shapiro-Wilk test. Employing the Levene test, equal variances were documented for the first three parameters and, therefore, significance of differences was evaluated using the t test. Subsequently, parameters of descriptive statistics were calculated (arithmetical mean, standard deviation, median value, minimum and maximum values). Results of microscopic image analysis were compared between the ACC and CCC independent groups of patients using the MannWhitney U test. The Wilcoxon test was used for nonparametric dependent data. Correlations between data rows were determined employing Spearman's rank correlation index. Differences and relationships were accepted to be statistically significant at the level of $p<0.05$.

\section{Results}

\section{Cellular localisation of human tryptase-positive} mast cells in gallbladder mucosa

Expression of tryptase-positive MCs was detected in all patients (100\%) of groups ACC and CCC. In all cases it involved mast cells with typical morphology ("fried eggs") (Fig. 1A). Tryptase-positive MCs were dispersed both in gallbladder lamina propria and within its epithelium (Fig. 1B).

\section{Cellular localisation of CD68-positive monocytes/macrophages in gallbladder mucosa}

Expression of the CD68 molecule was also demonstrated in gallbladders of all patients in both groups. The protein was seen in the cytoplasm, mainly in monocytes and macrophages but also in individual neutrophils 

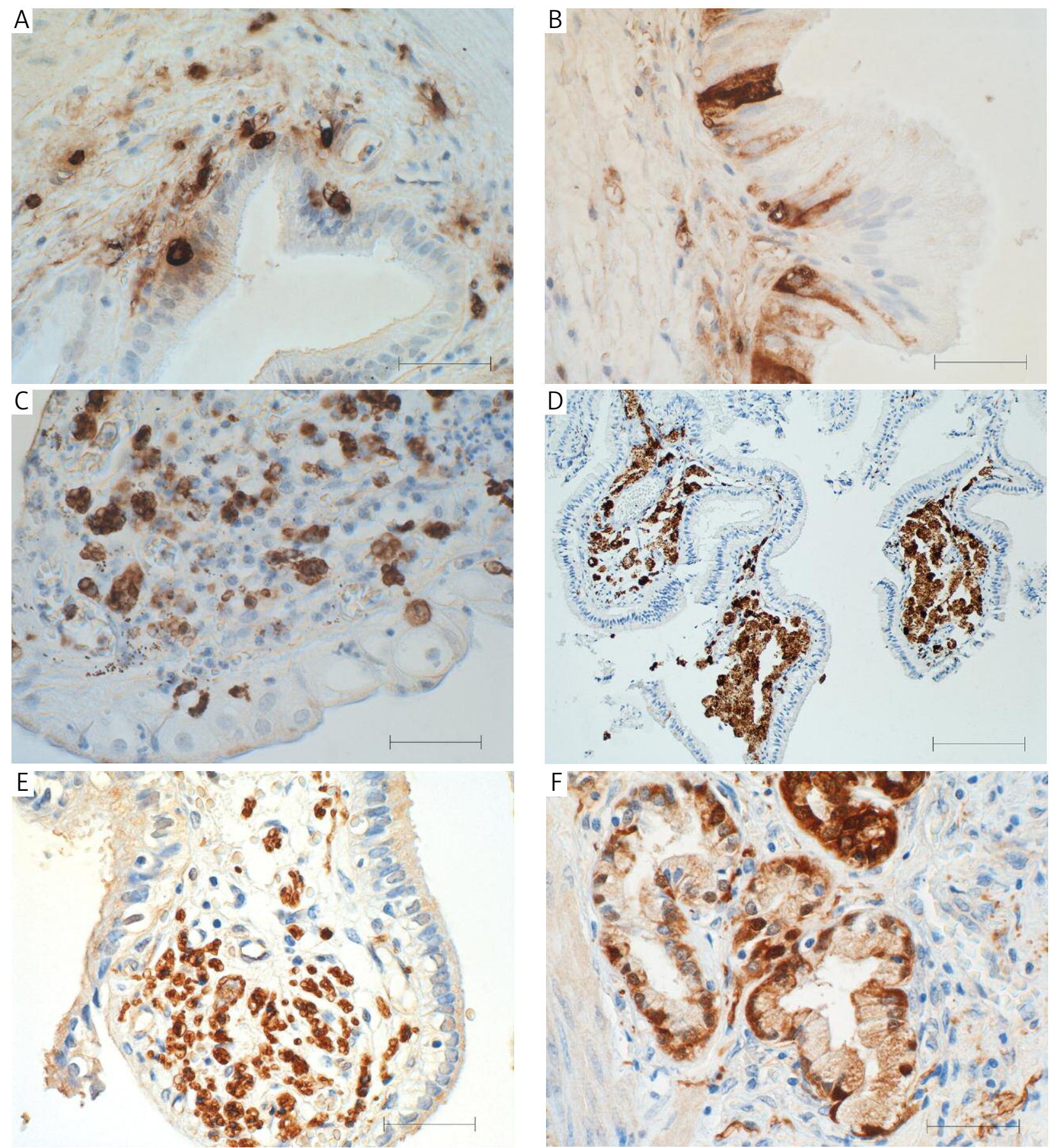

Fig. 1. Immunocytochemical localisation of cellular markers in gallbladder mucosa of patients with cholelithiasis: A) Tryptase-positive mast cells in lamina propria. B) Tryptase-positive mast cells located in gallbladder epithelium. C) CD68-positive macrophages among other inflammatory cells in lamina propria. D) CD68 expression in vast infiltrates of foamy cells (cholesterolosis). E) S100 protein-positive dendritic cells located in lamina propria under epithelium. F) S100 protein expression in tubulo-acinar glands in lamina propria. Occasionally nuclear localisation is present. Hematoxylin counterstained. Bar $=50 \mu \mathrm{m}(\mathrm{A}, \mathrm{B}, \mathrm{C}, \mathrm{E}, \mathrm{F}), 100 \mu \mathrm{m}$ (D)

(Fig. 1C). A particularly strong reaction was detected in clumps of enlarged foamy cells, occasionally very numerous under epithelium in patients of the CCC group (Fig. 1D). Vast infiltrates of foamy cells (cholesterolosis) were observed in 8/55 (14\%) patients from the CCC group.

\section{Cellular localisation of S100-positive dendritic cells in gallbladder mucosa}

Expression of the marker was also demonstrated in all patients of both groups. S100 protein-positive cells were manifested first of all in lamina propria (Fig. 1E). 

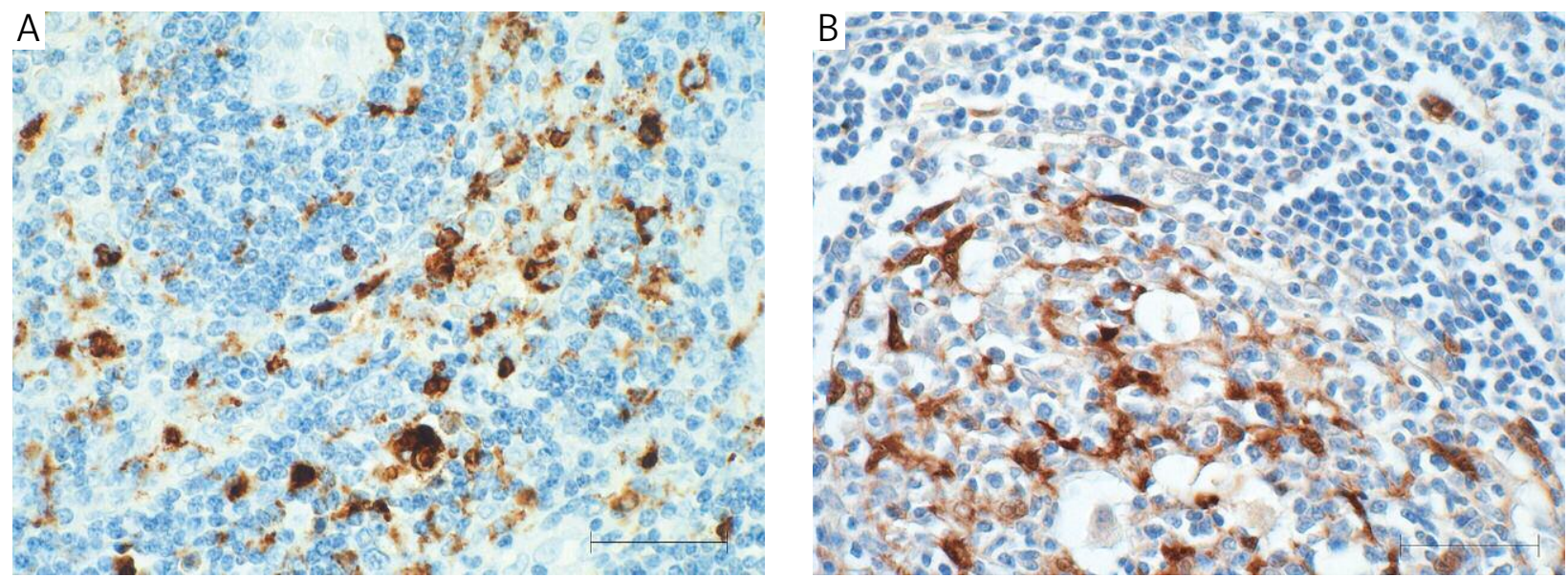

Fig. 2. Immunocytochemical localisation of cellular markers in reactive lymph nodes of patients with cholelithiasis (positive control): A) evident positive expression of CD68 in a great number of monocytes/macrophages; B) localisation of $\mathrm{S} 100$ protein in follicular dendritic cells. Hematoxylin counterstained. Bar $=50 \mu \mathrm{m}$

A positive reaction (including occasionally nuclear localisation) was also observed in tubulo-acinar glands (Fig. 1F).

In the positive control cellular expression of CD68 and $\mathrm{S} 100$ protein involved cells of lymph nodes (monocytes/macrophages and follicular DCs) (Fig. 2A and $2 \mathrm{~B}$ ).

\section{Morphometric evaluation of tryptase, CD68 and $\mathrm{S} 100$ protein in gallbladder mucosa}

Mean expression of tryptase in the CCC group manifested no significant differences as compared to the ACC group. Mean expression of CD68 in the ACC group was significantly higher than that in the CCC group. On the other hand, expression of S100 protein was significantly higher in the CCC group than in the ACC group (Table I, Fig. 3).

Upon comparison of expression involving the three marker proteins (Wilcoxon's test) within the ACC group, significant differences were disclosed in expression of tryptase (higher expression) and CD68 (higher expression) in comparison to expression of $\mathrm{S} 100$ protein.

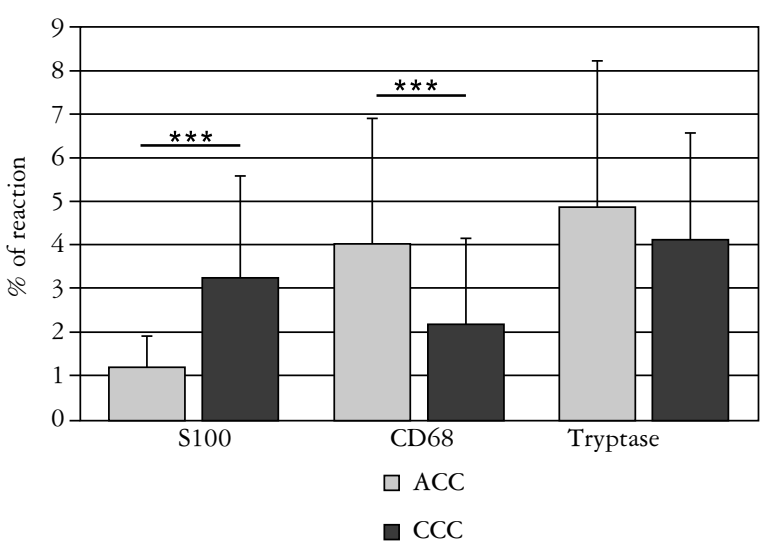

Fig. 3. Comparative expression of S100 protein, CD68 and tryptase between patients with acute and chronic calculous cholecystitis. $* * * \mathrm{p}$ (level of significance) value $<0.001$

In the group no significant differences were demonstrated between expression of tryptase and CD68. In patients with CCC significantly higher expression of tryptase and S100 protein was demonstrated, as com-

Table I. Comparative expression of tryptase, CD68 molecule, and S100 protein [area fraction (percentage) of the immunocytochemical reaction in gallbladder mucosa] as related to histopathological type of calculous cholecystitis

\begin{tabular}{lllllllll}
\hline Cellular Marker & GrouP & N & MEAN & SD & Min. & MaX. & P \\
\hline \multirow{2}{*}{ Tryptase } & ACC & 16 & 4.87 & 3.35 & 0.85 & 11.02 & 0.582 \\
\cline { 2 - 7 } & CCC & 55 & 4.13 & 2.45 & 0.39 & 9.94 & \\
\hline \multirow{2}{*}{ CD68 } & ACC & 16 & 4.04 & 2.86 & 1.90 & 12.31 & $<0.001$ \\
\cline { 2 - 7 } & CCC & 55 & 2.17 & 1.98 & 0.10 & 10.29 & \\
\hline
\end{tabular}

ACC - acute calculous cholecystitis, CCC - chronic calculous cholecystitis, $n$ - number of patients, $S D$ - standard deviation, min. - minimum, max. - maximum, $p$ - level of significance 
Table II. Comparison of mucosal expression (mean \% of immunocytochemical reaction \pm SD) involving the three cellular markers within acute and chronic type of calculous cholecystitis

\begin{tabular}{lccccc}
\hline MARKER & GrouP & \% OF IHC REACTION & MARKER & \% OF IHC REACTION & P \\
\hline \multirow{2}{*}{ Tryptase } & ACC & $4.87 \pm 3.35$ & CD68 & $4.04 \pm 2.86$ & 0.569 \\
\cline { 3 - 6 } & & & S100 & $1.18 \pm 0.74$ & $<0.001$ \\
\cline { 2 - 6 } & CCC & $4.13 \pm 2.45$ & CD68 & $2.17 \pm 1.98$ & $<0.001$ \\
\hline CD68 & & & S100 & $3.25 \pm 2.33$ & 0.069 \\
\hline & ACC & $4.04 \pm 2.86$ & S100 & $1.18 \pm 0.74$ & $<0.001$ \\
\hline
\end{tabular}

ACC - acute calculous cholecystitis, CCC - chronic calculous cholecystitis, IHC - immunocytochemical, SD - standard deviation, $p$ - level of significance

Table III. Spearman's rank correlation coefficients (r) between tryptase, CD68 and S100 protein expression (\% of reaction) in gallbladder mucosa and selected clinical data in patients with acute and chronic type of calculous cholecystitis

\begin{tabular}{|c|c|c|c|c|c|c|}
\hline \multirow[t]{2}{*}{ CLINICAL DATA } & \multicolumn{3}{|c|}{ ACC } & \multicolumn{3}{|c|}{ CCC } \\
\hline & TRYPTASE & CD68 & $\mathrm{S} 100$ & TRYPTASE & CD68 & S100 \\
\hline Age (years) & 0.109 & -0.024 & -0.372 & 0.238 & 0.258 & -0.015 \\
\hline BMI $\left(\mathrm{kg} / \mathrm{m}^{2}\right)$ & 0.429 & -0.072 & 0.068 & 0.224 & 0.198 & -0.002 \\
\hline Number of gallstones & 0.045 & 0.436 & -0.396 & 0.110 & -0.357 & 0.090 \\
\hline Width of gallbladder wall (mm) & 0.155 & 0.251 & 0.399 & -0.250 & 0.072 & 0.086 \\
\hline Grading* (G1) & 0.292 & 0.458 & -0.094 & 0.070 & 0.021 & 0.205 \\
\hline Grading* (G2) & 0.102 & 0.249 & 0.073 & -0.016 & 0.243 & 0.148 \\
\hline WBC $\left(\times 10^{9} / \mathrm{l}\right)$ & 0.082 & 0.030 & 0.464 & -0.052 & 0.156 & -0.041 \\
\hline
\end{tabular}

pared to expression of CD68. No significant differences were disclosed in intensities of tryptase and protein S100 expression (Table II).

\section{Correlations between expression of tryptase, CD68 and S100 protein and selected laboratory/clinical data of patients with cholelithiasis}

In neither patient group could significant correlations be documented between reciprocal expression of the markers (data not shown). Also no significant relationships were noted between expression of individual markers and intensity of inflammatory lesions (grading) in the gallbladder. In patients with CCC a weak negative correlation was disclosed between expression of CD68 and the number of gallstones (Table III).

\section{Discussion}

Mast cells (MCs) participate in the normal function of human mucosae and may play an active role in a group of diverse diseases [12]. In inflammatory infiltrates of the gallbladder, MCs and macrophages have been quantified using histological staining (e.g. toluidine blue), histochemical techniques (e.g. non-specific esterases) or immunohistochemistry (e.g. anti- $\alpha-1$ - antitrypsin) $[5,19,20]$. The pioneer investigations using these techniques were burdened with extensive difficulties of obtaining appropriate quality of histological pictures. Nevertheless, they demonstrated that esterase-positive cells (MCs) and $\alpha$-1-antitrypsin or $\alpha$ 1 -antichymotrypsin positive cells $(\mathrm{Mo} / \mathrm{Ma})$ are present in both normal and chronically inflamed gallbladder mucosa $[19,20\}$ and that esterase activity in gallbladder mucosa is significantly decreased in chronic cholecystitis [20]. Other investigators also observed less numerous MCs and macrophages in established chronic cholecystitis than in gallbladders with minimal inflammatory alterations [5].

In our study expression of tryptase was chosen for evaluation of MCs since as an $\alpha$-protryptase it is constitutively secreted from MCs and is a cell-specific protease. The protease can be measured in the serum, and thus serves as an indicator of the total number of MCs [32]. Its presence represents an exponent of MC degranulation [17]. In gallbladders with acute cholecystitis, mean expression of tryptase was slightly higher than that in the CCC group, but the difference was insignificant. The IHC reaction for the marker was well visible and provided the typical "fried eggs" pattern of MCs [18]. No correlation was detected between number of MCs and inflammatory activity in the gallbladder. Also, no significant relationships were revealed 
between the number of MCs in mucosal inflammatory infiltrates and the remaining clinical variables in the patients. We have not been able to confirm the literature data on augmented numbers of MCs in less intense lesions ("normal" tissue) of cholelithiasis in comparison with chronic cholecystitis [5], or their increased numbers as compared to the controls in chronic exacerbated and chronic sclerotic cholangitis in the patients with cholelithiasis [21]. Commenting on the differences, it should be added that for technical reasons we did not examine tryptase expression in "normal" gallbladder mucosa or "normal" choledochal wall, as it was performed in the quoted studies [5, 21]. In the studies of Hudson and Hopwood, other antibodies were applied for detection of MCs (rabbit antihuman $\alpha$-1-antitrypsin) [5], while Gulubova and Vodenicharov [21] examined cryostat sections while antibodies identical to those used by us were applied in a distinct dilution $(1: 50)$. In both cases a distinct quantitative evaluation of MCs was used (cells $\left./ \mathrm{mm}^{2}\right)[5,21]$. In our study we focused our attention on evaluation of MCs located exclusively within gallbladder mucosa and tryptase was applied as the basic marker of "mucosal" MCs [12, 15, 21]. It should be stressed that in our patients a tendency was detected toward increased expression of tryptase in the group with more intense inflammatory lesions (ACC), as compared to CCC, even if no correlation could be demonstrated with intensity of inflammatory lesions (grading). Possibly, by using a higher number of patients with ACC or examining tryptase-positive MCs in the entire wall of the inflamed gallbladders we might be able to demonstrate more correlations with grading or fibrosis of the organ. Examination of expression manifested by tryptase- and chymase-positive MCs in intralesional and perilesional skin lesions permitted potential involvement of MCs in pathogenesis of dermal tumours to be described [33]. No such studies are available in the case of gallbladder dysplastic and neoplastic lesions. The recent report demonstrated expression of two other immunohistochemical markers [DNA fragmentation factor-45 (DFF45) and thyroid transcription factor 1 (TTF-1)] in benign and malignant lesions of the gallbladder [34]. On the other hand, definition of the actual role played by MCs both in calculous cholecystitis and fibrosis of the organ as well as gallbladder carcinogenesis remains an open problem and requires continuation of the studies [17, 21, 22].

Our studies have demonstrated significantly more numerous CD68-positive macrophages in gallbladder mucosa in patients with acute cholecystitis, as compared to patients with CCC. On the other hand, only in CCC patients ( $14 \%$ of patients) were typical traits of cholesterolosis demonstrated, with presence of foamy macrophages, manifesting a pronounced positive reaction for CD68. The way in which the cells are generated remains unknown. Some authors suggest that scavenger receptor class $\mathrm{A}$ in macrophages is involved in the phagocytosis of $E$. coli, followed by foamy changes [9]. In xanthogranulomatous cholecystitis (XGC), characterized by a particularly pronounced accumulation of foamy cells among other inflammatory cells [35], it was confirmed that the vast majority of foamy cells were derived from monocytes/macrophages because they were positive for $\mathrm{CD} 68$ [8].

In our tissue material the CD68-positive cells included monocytes and macrophages of various size and individual neutrophils as well as markedly enlarged foamy cells (mainly in the CCC group). However, mean expression of CD68 antigen (and thus the number of macrophages) was higher in the ACC group, which seems to point to significant involvement of the cells in the first line of defence against microbes. Moreover, the cells may remove by phagocytosis the locally accumulated inflammatory cells. Thus, the role of macrophages is critical not only in the initiation of the inflammatory response, but also in the resolution of this process [36]. The increase in number of CD68-positive cells as compared to normal mucosa was also described in other active phases of inflammatory diseases in the alimentary tract (active Crohn's disease and ulcerative colitis) [37].

In the local inflammatory lesions of cholelithiasis both macrophages and dendritic cells (DCs) can function as antigen-presenting cells to propagate a local adaptive (antigen-specific) immune response [36].

Presence of S100 protein-positive DCs was demonstrated in $100 \%$ of gallbladders in both groups of patients. Using the spatial visualization technique higher expression of $\mathrm{S} 100$ protein was demonstrated in the CCC group than in the ACC group. No literature references are available with which the data could be compared. The descriptions related to gallbladder diseases are scarce and related to demonstration of S100 protein-positive Langerhans cells in a tumour of the organ [27]. Comparing expression of the studied cell markers, differences were also found within groups of patients. In mucosa of patients with ACC expression of tryptase (MCs) and CD68 (Mo/Ma) was similar and significantly higher than expression of $\mathrm{S} 100$ protein (DCs). This may point to active cooperation of MCs and macrophages in acute cholecystitis, involving mainly recruitment of neutrophils. In the mouse model the neutrophil chemoattractants, CXCL1/CXCL2, produced by both MCs and macrophages, were demonstrated to attract neutrophils to the site of acute inflammation [38]. The experiments permitted the authors to list a sequence of events, involving in the early phase of acute inflammation first the action of MCs located close to blood vessels, followed by neutrophil penetration of the tissue in a macrophage-dependent manner [38]. Other authors have also reported that mast cell-macrophage interactions alter inflammatory cytokine production and may contribute to chronic inflammation [11]. In the 
group with CCC expression of tryptase was higher than activity of CD68-positive macrophages, which might suggest other functions of the two cell populations in this phase of inflammation. Literature data include the suggestion of potential involvement of MCs, together with mediators produced by them, also in mucous hypersecretion in the gallbladder, which would promote lithogenesis [5]. In our patients with chronic cholecystitis expression of tryptase and S100 protein was similar and the two proteins manifested higher expression than that of CD68. As compared to ACC, expression of S100 protein was significantly higher in CCC. This might suggest more intense involvement of the cells in maintenance of the chronic stage of inflammation due to de novo formation of local lymphoid tissue [39]. Numerous lymphoid follicles were seen in almost 30\% of the patients with CCC described in our earlier paper [25]. In addition, interstitial DCs (S100 proteinpositive) are involved in stimulation of lymphocytes (mainly T lymphocytes) by constitutive production of the chemokines $[26,39]$. Dendritic cells are functionally linked to several aspects of immune tolerance and autoimmunity but our results do not permit us to comment on such literature data [39]. In order to better recognise the role of locally accumulated DCs studies should continue, aimed first of all at definition of the mechanisms due to which DCs accumulate in mucosa of gallbladders with calculous cholecystitis and at evaluation of the quantity of their precursors, e.g., CD34positive dendritic interstitial cells [26].

\section{Summary and conclusions}

The new element in this study involves evaluation of immunocytochemical expression using the modern technique of spatial visualization applied to typical markers of cells which play key roles in inflammatory reactions, the acute (ACC) and chronic type of cholecystitis (CCC) in patients of various age. In gallbladder mucosa of patients with ACC or CCC, similar numbers of mast cells were observed. Expression of CD68 prevailed in ACC and expression of S100 in CCC, which points to distinct functions of the studied cell types in the non-specific immune response accompanying calculous cholecystitis.

The spatial visualization technique allowed for a credible quantitative appraisal of marker expression, typical of MCs, Mo/Ma and DCs in gallbladder mucosa of patients with ACC or CCC. For the first time mucosal expression of S100 protein-positive DCs was characterized in calculous cholecystitis. The results point to distinct functions of studied cell types in the non-specific immune response associated with ACC and CCC.

The first author (MS) received a grant within Subaction 8.2.2. "Regional Strategies of Innovation", Action 8.2
"Transfer of knowledge", Priority VIII "Regional Staff of Management", Operative Programme of Human Capital, cofinanced by the European Social Fund of the European Union and by the state budget.

\section{References}

1. Rosai J. Gallbladder and extrahepatic bile ducts. In: Rosai and Ackerman's Surgical Pathology. 9th ed. Rosai J (ed.). Mosby, Edinburgh, London, New York, Oxford, Philadelphia, St. Louis, Sydney, Toronto 2004; 1035-1055.

2. Pitt HA. Hepato-pancreato-biliary fat: the good, the bad and the ugly. HPB (Oxford) 2007; 9: 92-97.

3. Baig SJ, Biswas S, Das S, et al. Histopathological changes in gallbladder mucosa in cholelithiasis: correlation with chemical composition of gallstones. Trop Gastroenterol 2002; 23: 25-27.

4. English M, Hopwood D. Lipid in the human gallbladder mucosa. A histochemical study by light and electron microscopy. J Pathol 1985; 146: 333-336.

5. Hudson I, Hopwood D. Macrophages and mast cells in chronic cholecystitis and "normal" gall bladders. J Clin Pathol 1986; 39: 1082-1087.

6. Franco V, Aragona F, Genova G, et al. Xanthogranulomatous cholecystitis. Histopathological study and classification. Pathol Res Pract 1990; 186: 383-390.

7. Mori M, Watanabe M, Sakuma M, Tsutsumi Y. Infectious etiology of xanthogranulomatous cholecystitis: immunohistochemical identification of bacterial antigens in the xanthogranulomatous lesions. Pathol Int 1999; 49: 849-852.

8. Nakashiro H, Haraoka S, Fujiwara K, et al. Xanthogranulomatous cholecystitis. Cell composition and a possible pathogenic role of cell-mediated immunity. Pathol Res Pract 1995; 191 : 1078-1086.

9. Sawada S, Harada K, Isse K, et al. Involvement of Escherichia coli in pathogenesis of xanthogranulomatous cholecystitis with scavenger receptor class A and CXCL16-CXCR6 interaction. Pathol Int 2007; 57: 652-663.

10. Dabbs DJ. Eosinophilic and lymphoeosinophilic cholecystitis. Am J Surg Pathol 1993; 17: 497-501.

11. Metcalfe DD, Baram D, Mekori YA. Mast cells. Physiol Rev 1997; 77: 1033-1079.

12. Krishnaswamy G, Kelley J, Johnson D, et al. The human mast cells: functions in physiology and disease. Front Biosci 2001; 6: D1109-D1127.

13. Welle M. Development, significance, and heterogeneity of mast cells with particular regard to the mast cell-specific proteases chymase and tryptase. J Leukoc Biol 1997; 61: 233-245.

14. Bradding P, Roberts JA, Britten KM, et al. Interleukin-4, -5, and -6 and tumor necrosis factor-alpha in normal and asthmatic airways: evidence for the human mast cell as a source of these cytokines. Am J Respir Cell Mol Biol 1994; 10: 471-480.

15. Bradding P, Okayama Y, Howarth PH, et al. Heterogeneity of human mast cells based on cytokine content. J Immunol 1995; 155: 297-307.

16. Bischoff SC, Lorentz A, Schwengberg S, et al. Mast cells are an important cellular source of tumour necrosis factor $\alpha$ in human intestinal tissue. Gut 1999; 44: 643-652.

17. He SH. Key role of mast cells and their major secretory products in inflammatory bowel disease. World J Gastroenterol 2004; 10: 309-318.

18. Hermanowicz A, Debek W, Dzienis-Koronkiewicz E, Chyczewski L. Topography and morphometry of intestinal mast cells in children with Hirschsprung's disease. Folia Histochem Cytobiol 2008; 46: 65-68.

19. Aroni K, Kittas C, Papadimitriou CS, Papacharalampous NX. An immunocytochemical study of the distribution of lysozyme, 
a1-antitrypsin and a1-antichymotrypsin in the normal and pathological gall bladder. Virchows Arch A Pathol Anat Histopathol 1984; 403: 281-289.

20. Kouroumalis E, Hopwood D, Ross PE, Bouchier IA. Human gallbladder epithelium: non-specific esterases in cholecystitis. J Pathol 1984; 142: 151-159.

21. Gulubova M, Vodenicharov A. Structural examination of tryptase-, and VIP-positive mast cells in the common bile duct of patients with lithiasis. Acta Histochem 2001; 103: 437-452.

22. Friesen CA, Neilan N, Daniel JF, et al. Mast cell activation and clinical outcome in pediatric cholelithiasis and biliary dyskinesia. BMC Res Notes 2011; 4: 322-329.

23. Fox H, Mainwaring AR. Eosinophilic infiltration of the gallbladder. Gastroenterology 1972; 63: 1049-1052.

24. Kleinschmidt S, Meneses F, Nolte I, Hewicker-Trautwein M. Characterization of mast cell numbers and subtypes in biopsies from gastrointestinal tract of dogs with lymphocytic-plasmacytic or eosinophilic gastroenterocolitis. Vet Immunol Immunopathol 2007; 120: 80-92.

25. Kasprzak A, Malkowski W, Biczysko W, et al. Histological alterations of gallbladder mucosa and selected clinical data in young patients with symptomatic gallstones. Pol J Pathol 2011; 62: 41-49.

26. Papadas T, Batistatou A, Ravazoula P, et al. S-100 proteinpositive dendritic cells and CD34-positive dendritic interstitial cells in palatine tonsils. Eur Arch Otorhinolaryngol 2001; 258: 243-245.

27. Zhao G, Luo M, Wu ZY, et al. Langerhans cell sarcoma involving gallbladder and peritoneal lymph nodes: a case report. Int J Surg Pathol 2009; 17: 347-353.

28. Szmyt M, Kasprzak A, Malkowski W, et al. Tissue expression of S100 proteins in gallbladder mucosa of the patients with calculous cholecystitis. Folia Histochem Cytobiol 2013; 51: 1-8.

29. Sabattini E, Bisgaard K, Ascani S, et al. The EnVision + + system: a new immunohistochemical method for diagnostics and research. Critical comparison with the APAAP, ChemMate, CSA, LABC, and SABC techniques. J Clin Pathol 1998; 51: 506-511.

30. Kaczmarek E, Strzelczyk R. From two to three-dimensional visualisation of structures in light and confocal microscopy - applications for biomedical studies. In: Mendez-Vilas A, LabajosBroncano L (eds). $1^{\text {st }}$ ed. Current issues on multidisciplinary microscopy research and education. FORMATEX microscopy book series no. II Formatex Research Centre, Badajoz 2005; 289295.

31. Kasprzak A, Malkowski W, Seraszek A, et al. Cytokeratin 8 and 18 tissue expression in gallbladder mucosa of patients with cholelithiasis. Pol J Pathol 2011; 4: 241-249.

32. Sperr WR, Jordan JH, Fiegl M, et al. Serum tryptase level in patients with mastocytosis: correlation with mast cell burden and implication for defining the category of disease. Int Arch Allergy Immunol 2002; 128: 136-141.

33. Dyduch G, Okoń K, Pescarini E. Mast cells in melanocytic skin lesions. An immunocytochemical and quantitative study. Pol J Pathol 2011; 3: 139-144.

34. Yuan Y, Yang ZL, Zou Q, et al. Clinicopathological significance of DNA fragmentation factor 45 and thyroid transcription factor 1 expression in benign and malignant lesions of the gallbladder. Pol J Pathol 2013; 64: 44-51.

35. Cozzutto C, Carbone A. The xanthogranulomatous process. Xanthogranulomatous inflammation. Pathol Res Pract 1988; 183: 395-402.

36. Zhang X, Mosser DM. Macrophage activation by endogenous danger signals. J Pathol 2008; 214: 161-178.

37. Rugtveit J, Brandtzaeg P, Halstensen TS, et al. Increased macrophage subset in inflammatory bowel disease: apparent recruitment from peripheral blood monocytes. Gut 1994; 35: 669674 .

38. De Filippo K, Dudeck A, Hasenberg M, et al. Mast cell and macrophage chemokines CXCL1/CXCL2 control the early stage of neutrophil recruitment during tissue inflammation. Blood 2013; 121: 4930-4937.

39. Sallusto F, Lanzavecchia A. Mobilizing dendritic cells for tolerance, priming, and chronic inflammation. J Exp Med 1999; 189: 611614.

\section{Address for correspondence}

\section{Prof. Aldona Anna Kasprzak, MD, PhD}

Chair and Department of Histology and Embryology

Poznan University of Medical Sciences

Swiecickiego 6

60-781 Poznan, Poland

tel. +48618546455

fax +48618546440

e-mail: akasprza@ump.edu.pl 